\title{
Mutations in voltage-gated sodium channels from pyrethroid resistant salmon lice (Lepeophtheirus salmonis)
}

Running title: Kdr mutations in L. salmonis

\author{
Greta Carmona-Antoñanzas ${ }^{1,2}$, Kari O Helgesen ${ }^{3}$, Joseph L. Humble ${ }^{4}$, Claudia \\ Tschesche $^{5}$, Marit J Bakke ${ }^{6}$, Louise Gamble ${ }^{7}$, Michaël Bekaert ${ }^{8}$, David I. Bassett ${ }^{9}$, Tor E \\ Horsberg $^{10}$, James E Bron ${ }^{11}$ and Armin Sturm ${ }^{12^{*}}$
}
${ }^{1}$ Institute of Aquaculture, Faculty of Natural Sciences, University of Stirling, Stirling, FK9 4LA, United Kingdom

${ }^{2}$ Imegen, Parc Científic de la Universitat de València, 46980 Paterna (Valencia, Spain), email: greta.carmona@imegen.es

${ }^{3}$ Norwegian Veterinary Institute, Department of Epidemiology, Ullevålsveien 68, Pb 750 Sentrum, N-0106 Oslo, Norway, email: kari.helgesen@vetinst.no

${ }^{4}$ Institute of Aquaculture, Faculty of Natural Sciences, University of Stirling, Stirling, FK9 4LA, United Kingdom, email: joeyhumble@gmail.com

${ }^{5}$ Institute of Aquaculture, Faculty of Natural Sciences, University of Stirling, Stirling, FK9 4LA, United Kingdom, email: claudia.tschesche@stir.ac.uk

${ }^{6}$ Norwegian University of Life Sciences, Faculty of Veterinary Medicine, Sea Lice

Research Centre, Oslo, Norway, email: marit.bakke@nmbu.no

${ }^{7}$ Institute of Aquaculture, Faculty of Natural Sciences, University of Stirling, Stirling, FK9 4LA, United Kingdom, email: I.gamble82@gmail.com

${ }^{8}$ Institute of Aquaculture, Faculty of Natural Sciences, University of Stirling, Stirling, FK9 4LA, United Kingdom, email: michael.bekaert@stir.ac.uk

${ }^{9}$ Institute of Aquaculture, Faculty of Natural Sciences, University of Stirling, Stirling, FK9 4LA, United Kingdom, email: david.bassett@stir.ac.uk

${ }^{10}$ Norwegian University of Life Sciences, Faculty of Veterinary Medicine, Sea Lice

This article has been accepted for publication and undergone full peer review but has not been through the copyediting, typesetting, pagination and proofreading process, which may lead to differences between this version and the Version of Record. Please cite this article as doi: 10.1002/ps.5151 
Research Centre, Oslo, Norway, email: tor.e.horsberg@nmbu.no

${ }^{11}$ Institute of Aquaculture, Faculty of Natural Sciences, University of Stirling, Stirling, FK9 4LA, United Kingdom, email: j.e.bron@stir.ac.uk

${ }^{12}$ Institute of Aquaculture, Faculty of Natural Sciences, University of Stirling, Stirling, FK9 4LA, United Kingdom, email: armin.sturm@stir.ac.uk

*corresponding author 


\section{Abstract}

Background: Parasitic salmon lice (Lepeophtheirus salmonis) cause high economic losses in Atlantic salmon farming. Pyrethroids, which block arthropod voltage-gated sodium channels $\left(\mathrm{Na}_{v} 1\right)$, are used for salmon delousing. However, pyrethroid resistance is common in $L$. salmonis. The present study characterised $\mathrm{Na}_{v} 1$ homologues in $L$. salmonis in order to identify channel mutations associated to resistance, called kdr (knockdown) mutations.

Results: Genome scans identified three L. salmonis $N a_{v} 1$ homologues, $L s N a_{v} 1.1, L s N a_{v} 1.2$ and $L s N a_{v} 1$.3. Arthropod $k d r$ mutations map to specific $\mathrm{Na}_{v} 1$ regions within domains DI-III, namely segments S5 and S6 and the linker helix connecting S4 and S5. The above channel regions were amplified by RT-PCR and sequenced in deltamethrin-susceptible and deltamethrin-resistant $L$. salmonis. While $L s N a_{v} 1.1$ and $L s N a_{v} 1.2$ lacked nucleotide polymorphisms showing association to resistance, $L s N a_{v} 1.3$ showed a non-synonymous mutation in S5 of DIl occurring in deltamethrin-resistant parasites. The mutation is homologous to a previously described kdr mutation (I936V, numbering according to Musca domestica Vssc1) and was present in two pyrethroid-resistant L. salmonis strains (allele frequencies of 0.800 and 0.357 ), but absent in two pyrethroid-susceptible strains.

Conclusions: The present study indicates that a kdr-mutation in $L s N a_{V} 1.3$ may contribute to deltamethrin resistance in L. salmonis. 


\section{Keywords}

Deltamethrin, sea lice, sodium channel, resistance

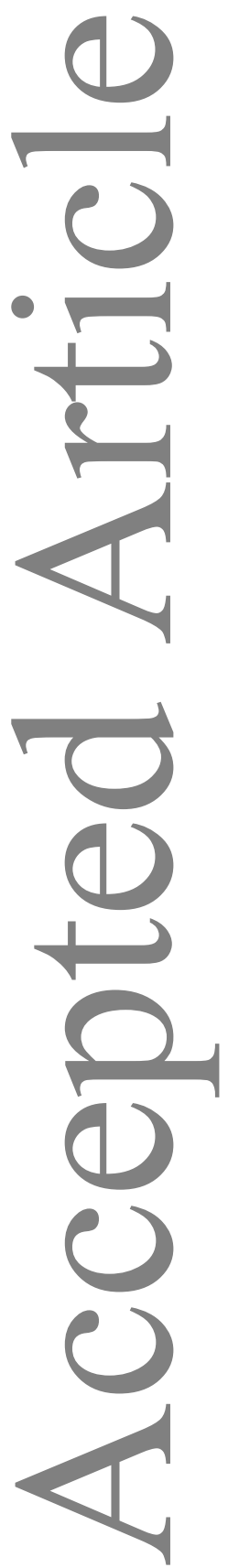




\section{Introduction}

Voltage-gated sodium channels play essential roles in the initiation and propagation of action potentials in neurons, myocytes and other electrically excitable cells ${ }^{1}$, and are the pharmacological targets of different types of natural neurotoxins and synthetic chemicals interfering with channel gating to disrupt neurotransmission ${ }^{2}$. Such compounds include toxins from pufferfish, scorpions and cnidarians, local anaesthetics, and insecticides ${ }^{2-4}$. The widespread use of insecticides interacting with sodium channels, namely DDT and the pyrethroids, has led to the evolution of resistance in arthropod populations under selection pressure. Point mutations in voltage-gated sodium channels are a common mechanism of pyrethroid resistance ${ }^{5}$.

Pyrethroids are synthetic analogues to botanical insecticides of the class of pyrethrins, which are produced by flowers of the genus Chrysanthemum. A number of distinct structural traits of pyrethroids cause their greater stability and efficacy as pest control agents compared to pyrethrins ${ }^{6}$. Pyrethroids are widely used to control phytophagous insects, human-disease vectors and animal parasites. Pyrethroids accounted for $17 \%$ of global insecticides sales in 2013, which makes them the second most important insecticide class after neonicotinoids ${ }^{6,7}$. However, their efficacy to control arthropod pests is challenged by the evolution of pyrethroid resistance ${ }^{8,9}$, which constitutes a major threat to food security and human health, and can lead to extensive economic losses ${ }^{10}$.

The toxicity of pyrethroids is based on their blocking of neuronal voltage-gated sodium channels, which involves interaction with the pore-forming $\alpha$-subunit of the channel called $\mathrm{Na}_{v}{ }^{4,11} . \mathrm{Na}_{v}$ consists of four internally homologous domains DI-IV, which are arranged symmetrically to form a central pore ${ }^{2}$. Knockdown resistance $(\mathrm{kdr})$ is a key mechanism of pyrethroid resistance in arthropods and results from non-synonymous point mutations of $\mathrm{Na}_{\mathrm{v}}$, reducing the channel's sensitivity to pyrethroids and usually conferring cross-resistance to $\mathrm{DDT}^{2,4,12}$. Alternatively, pyrethroid resistance can result from genetic changes that lead to the enhanced expression of biochemical detoxification pathways, often involving cytochromes P450 (CYPs) and/or glutathione-S-transferases (GSTs) ${ }^{9,13,14}$.

Since the mid-1990s, the pyrethroids cypermethrin and deltamethrin have been licensed as veterinary medicines to treat farmed Atlantic salmon (Salmo salar Linnaeus, 1758) and rainbow trout (Oncorhynchus mykiss Walbaum, 1792) suffering from infections by sea lice (Copepoda: Caligidae) ${ }^{15,16}$. Sea lice are ectoparasites feeding on the mucus, skin and blood of host fish. At high infection densities, sea louse infections can give rise to skin lesions, osmoregulatory imbalances, growth and immune suppression, secondary infections and, if untreated, potentially death ${ }^{16}$. In the Northern hemisphere, most caligid infections of farmed 
salmon involve the salmon louse (Lepeophtheirus salmonis, (Krøyer, 1837)). Two allopatric subspecies of $L$. salmonis exist, of which $L$. salmonis salmonis (Krøyer, 1837) inhabits the North Atlantic while L. salmonis oncorhynchi Skern-Mauritzen, Torrissen \& Glover, 2014 is found in the North-East Pacific ${ }^{17}$. Infection by the smaller species Caligus elongatus Nordmann, 1832 also occurs in the Northern hemisphere. In Chile, the sea louse species Caligus rogercresseyi Boxshall \& Bravo, 2000 is a significant pathogen in commercial salmon production ${ }^{18}$.

Only a limited range of veterinary drugs are available as licensed salmon delousing treatments ${ }^{19}$. The repeated use of a restricted range of anti-parasitic drugs can favour the evolution of resistance in the parasite ${ }^{20}$. Resistance to pyrethroids has been reported for both $L$. salmonis and $C$. rogercresseyi ${ }^{21,22}$ and resistance to pyrethroids in $L$. salmonis is currently common in the North Atlantic ${ }^{20}$. The mechanism of pyrethroid resistance in $L$. salmonis is at present unknown. In 2005, a kdr-type mutation in the L. salmonis $\mathrm{Na}_{\mathrm{v}} 1$ homologue was proposed as a major determinant of pyrethroid resistance in this fish parasite ${ }^{23}$; however, no later studies providing supporting evidence for this mechanism exist. Similarly, the involvement of cytochrome P450 monooxygenases in the detoxification of pyrethroids in $L$. salmonis has been demonstrated ${ }^{24}$, but its relation to resistance is unknown. Recently, it has been demonstrated that pyrethroid resistance is inherited maternally in L. salmonis ${ }^{25,26}$. While the results suggested that both mitochondrial and nuclear genetic determinants contributed to the resistance, the molecular mechanism causing resistance remains to be identified.

The aim of the present study was to assess potential roles for $\mathrm{Na}_{v}$ in determining the resistance of $L$. salmonis against pyrethroids by comparing nucleotide sequences and expression levels of $\mathrm{Na}_{v} 1$ homologues between two well characterised laboratory maintained strains of the parasite, the deltamethrin-resistant strain loA-02 and the deltamethrinsusceptible strain loA-00. The expression of candidate single nucleotide polymorphisms identified by this approach was further investigated using allele-specific genotyping assays, taking into account the above and two further parasite strains, one of which was deltamethrin resistant.

\section{Materials and Methods}

\subsection{Ethics statement}

All research projects involving the Institute of Aquaculture (IoA) are subjected to a thorough Ethical Review Process prior to any work being approved. All projects with loA participation 
are required to be submitted to the loA Ethical Committee for approval, irrespective of where experimentation will be carried out. This procedure ensures all ethical issues are addressed before an experiment can be initiated. The present research was assessed by the loA Ethical Review Committee and passed the Ethical Review Process of the University of Stirling (Project ID ASPA10/2013). Laboratory infections of Atlantic salmon with L. salmonis were carried out under UK Home Office project license 645 PPL 60/4522.

\subsection{Lepeophtheirus salmonis strains and husbandry}

The laboratory-maintained strains of $L$. salmonis used in this study were established from egg strings collected from Scottish salmon production sites ${ }^{26,27}$. No drug selection has been applied during the isolation or maintenance of the strains. Between their isolation and the year 2015, in which the experiments described in this report were carried out, strains have shown stable drug susceptibility profiles in bioassays (data not shown). Strain loA-00 was established in 2003 from an isolate originating in the Firth of Clyde and is susceptible to all current delousing agents including deltamethrin. Strain loA-01, derived in 2008 from material collected in Sutherland, is susceptible to deltamethrin ${ }^{26}$ but resistant to emamectin benzoate ${ }^{27}$. Strains loA-02 and loA-03, which were established in 2011 and 2012, respectively, from the Shetland Islands and Sutherland, are resistant to emamectin benzoate and deltamethrin 26,27 . In bioassays involving $30 \mathrm{~min}$ of pesticide exposure and $24 \mathrm{~h}$ of recovery, the following deltamethrin median effective concentrations $\left(E_{50} \mathrm{~S}\right)$, followed by $95 \%$ confidence limits, were determined in a previous study ${ }^{26}$ : IoA-00: $0.28 \mu \mathrm{g} \mathrm{L}^{-1}\left(0.23-0.36 \mu \mathrm{g} \mathrm{L}^{-1}\right)$, IoA-01: $0.36 \mu \mathrm{g} \mathrm{L}^{-1}\left(0.26-0.46 \mu \mathrm{g} \mathrm{L}^{-1}\right)$, loA-02: $40.1 \mu \mathrm{g} \mathrm{L}^{-1}\left(22.1\right.$ - 158.9 $\left.\mu \mathrm{g} \mathrm{L}^{-1}\right)$, loA-03: $>2.0 \mu \mathrm{g} \mathrm{L}$ 1 . Under culture, strains have been maintained under identical conditions as described in detail elsewhere ${ }^{28}$. In brief, parasites were maintained on Atlantic salmon hosts kept in circular tanks supplied with fresh seawater at ambient temperature, using a photoperiod corresponding to natural day length. To propagate cultures, egg strings were obtained from gravid females, hatched and allowed to develop to copepodids, which were used to infect naïve host fish. Infection rates were maintained at levels that were unlikely to compromise fish welfare. Prior to the collection of $L$. salmonis from hosts, host fish were euthanised under a UK Home Office approved Schedule 1 method. All laboratory infections were carried out under UK Home Office licence and appropriate veterinary supervision.

\subsection{Identification of $\mathrm{Na}_{\mathrm{v}}$ homologues}

To identify L. salmonis $\mathrm{Na}_{v}$ homologues, BLASTn searches were performed on the $L$. salmonis salmonis genome assembly (ftp://ftp.ensemblgenomes.org/pub/metazoa/release36/fasta/lepeophtheirus salmonis) using the $\mathrm{Na}_{v} 1 \mathrm{cDNA}$ sequence of the marine copepod Acartia hudsonica Pinhey, 1926 [GenBank: KP985762] as the query ${ }^{29}$. Three hits of high 
homology scores $\left(\mathrm{E}\right.$-value $<10^{-4}$ ) were obtained, suggesting that three loci encoding $\mathrm{Na}_{\mathrm{v}}$ homologues exist in L. salmonis (Supporting information: Table S1). Using ensemble gene models for the three loci, further searches were conducted to identify homologous sequences in other $L$. salmonis genome assemblies (GenBank accession numbers GCA_001005205.1 and GCA_000181255.2) and a transcriptome assembly of the species (EBI ENA reference ERS237607) (Supporting information: Table S1) ${ }^{30}$.

\subsection{Phylogenetic analyses}

Phylogenetic analyses of $L$. salmonis $\mathrm{Na}_{v}$ protein sequences took into account sodium channels from a number of arthropods, including several crustaceans and the planktonic copepod A. hudsonica ${ }^{29}$. $\mathrm{Na}_{\mathrm{v}}$ channels are formed by four homologous domains (I-IV) responsible for channel opening, ion selectivity and voltage sensing linked by highly variable intracellular loops (Figure 1) ${ }^{31}$. Full and partial protein sequences of 21 invertebrate species were aligned using the programme Clustal Omega ${ }^{32}$ and then subjected to phylogenetic analysis using the RAxML package ${ }^{33}$. For the planktonic copepod Calanus finmarchicus (Gunnerus, 1770), one full-length CDS was included (GenBank: GAXK01037395.1) and the partial sequences of two paralogous $\mathrm{Na}_{v} 1$ genes ${ }^{34}$, since full-length sequences were not available. The phylogenetic trees were constructed from the amino acid sequences of domain I using a maximum likelihood method implementing the WAT model with 1000 bootstrapping iterations.

\subsection{L. salmonis cDNA synthesis}

To isolate total RNA, individual parasites (adult males or preadult II females) were homogenised in TRI Reagent (Sigma-Aldrich, UK) using a Mini-Beadbeater-24 (BioSpec Products). RNA extraction followed the TRI Reagent manufacturer's instructions. The quantity and integrity of isolated RNA was determined by agarose gel electrophoresis and spectrophotometry (Nanodrop ND-1000, Thermo Scientific). Two micrograms of total RNA were reverse transcribed using the Verso cDNA synthesis kit (Thermo Scientific, UK), employing $7.5 \mu \mathrm{M}$ random hexamers and $2.5 \mu \mathrm{M}$ oligo(dT). The resulting cDNA was diluted 20 -fold with nuclease-free water and stored at $-70^{\circ} \mathrm{C}$ prior to further use.

\section{6 $\mathrm{Na}_{\mathrm{v}}$ amplification and sequencing}

To confirm the sequences of the three $L$. salmonis $\mathrm{Na}_{v} 1$ homologues identified in silico, and to conduct sequence comparisons between pyrethroid susceptible and resistant parasites, channel cDNAs were amplified from $L$. salmonis strains loA-00 and loA-02 by RT-PCR. Strain loA-00 has been consistently deltamethrin-susceptible in all bioassays conducted between 2011 and 2015 (i.e., 100\% of observed effect after exposure to $1 \mathrm{\mu g} \mathrm{L}^{-1}$ deltamethrin, data not shown), thus parasites from this strain were used without selection. 
Strain loA-02 parasites were variable in their responses at deltamethrin levels greater than $10 \mu \mathrm{L} \mathrm{L}^{-1}$. For the analysis of channel sequences, highly resistant loA-02 L. salmonis were used, which had failed to show behavioural effects after exposures to $40 \mathrm{\mu g} \mathrm{L}^{-1}$ deltamethrin in bioassays (see below). In both strains, three adult male and three preadult-Il female parasites were subjected to sequence analysis, but due to failure of some sequencing reactions, data are only available for five parasites per strain for some channel regions. Specific nested PCR oligonucleotide primers designed to target the L. salmonis sodium channels were used to amplify domains DI, DII and DIII of each $\mathrm{Na}_{v} 1$ (Supporting information, Table S2) ${ }^{2}$. PCRs were performed using $1 \mu \mathrm{l}$ of diluted cDNA synthesised as described above with $0.3 \mu \mathrm{M}$ of each oligonucleotide, $5 \mathrm{mM}$ of each dNTP and 5 units of Takara LA Taq Hot Start polymerase (Takara Bio, USA) in a total volume of $50 \mu \mathrm{L}$. Reactions were run in a Mastercycler RealPlex (Biometra, UK). PCR conditions consisted of a denaturing step at $96^{\circ} \mathrm{C}$ for $2 \mathrm{~min}$, followed by 30 cycles of denaturation at $96^{\circ} \mathrm{C}$ for 30 sec, annealing at the specified temperature (Additional file 2, Table S2) and extension at $72^{\circ} \mathrm{C}$ for 1 min $\mathrm{kb}^{-1}$ expected amplicon size. PCR products were purified (QIAquick PCR Purification Kit, QIAGEN, USA) and sequenced using capillary sequencing technology (Macrogen, Netherlands) and appropriate primers to obtain sequences covering sequences encoding S1 to S6 of domains I, II and III (Supporting information, Table S2). Each sequence generated was manually assessed and trimmed, removing low quality regions. Subsequently, sequences of individual parasites were aligned against the reference loA-00 consensus sequences using BioEdit (v7.05) to identify nucleotide polymorphisms.

\subsection{Genotyping of single nucleotide (SNP) alleles}

For selected SNPs, genotypes of parasites of four strains (IoA-00, loA-01, loA-02, IoA-03; 8 males and 8 females per strain) were determined through allele-specific PCR assays using universal fluorescence energy transfer (FRET) probes $^{35}$. Genomic DNA was extracted from L. salmonis specimens conserved in absolute ethanol using the method of Blanquer ${ }^{36}$, with details having been reported elsewhere ${ }^{26}$. Each SNP assay involved one common primer and two allele specific primers (Table S2). Reaction mixtures (final volume $10 \mu \mathrm{L}$ ) contained $25 \mathrm{ng}$ gDNA (lacking in no-template controls), $166 \mathrm{nM}$ of each allele specific primer, $276 \mathrm{nM}$ of the common primer and $1 \mathrm{X}$ KASP $®$ master mix (LGC Genomics, UK). Assays were run in 96-well plates in a Quantica PCR thermocycler (Bibby Scientific, UK). The PCR programme comprised an initial denaturation / activation step $\left(94^{\circ} \mathrm{C}\right.$ for $\left.15 \mathrm{~min}\right)$, ten cycles of a two-step touchdown programme (denaturation at $94^{\circ} \mathrm{C}$ for $20 \mathrm{sec}$, annealing at $65-57^{\circ} \mathrm{C}$ for $60 \mathrm{sec}$, with a decrease in annealing temperature of $0.6^{\circ} \mathrm{C}$ per cycle) and 33 cycles of a regular two-step programme $\left(94^{\circ} \mathrm{C}\right.$ for $20 \mathrm{sec}, 57^{\circ} \mathrm{C}$ for $\left.60 \mathrm{sec}\right)$. Genotypes of individuals 
were derived based on the observed relative strength of signals of the two allele-specific fluorophores FAM and HEX.

\subsection{Deltamethrin exposures}

The effects of deltamethrin exposures on $\mathrm{Na}_{v}$ transcript expression were tested in salmon lice of strains loA-00 (drug-susceptible) and loA-02 (pyrethroid resistant). Experimental exposures of $L$. salmonis were at two levels, $0.05 \mu \mathrm{g} \mathrm{L}^{-1}$ deltamethrin and $2 \mu \mathrm{g} \mathrm{L}^{-1}$ deltamethrin. Control groups received seawater containing $0.05 \%(\mathrm{v} / \mathrm{v})$ vehicle $\mathrm{PEG}_{300}$ (polyethylene glycol, $M_{n}=300$ ). Parasites were collected from host fish, randomly allocated to $300 \mathrm{~mL}$ crystallising dishes containing $100 \mathrm{~mL}$ of seawater at $12^{\circ} \mathrm{C}$ and incubated for 1 to $3 \mathrm{~h}$ before the start of exposures. A stock solution of $0.5 \mathrm{mg} \mathrm{mL}^{-1}$ deltamethrin (Pestanal $\AA$ analytical standard, Sigma-Aldrich, UK) was prepared in $\mathrm{PEG}_{300}$ (polyethylene glycol, $\mathrm{M}_{\mathrm{n}}=$ 300 ) and further diluted in seawater. Duplicate dishes, each containing 5 adult males and 5 preadult II females, were used for deltamethrin and control treatments. After 30 min of exposure at $12^{\circ} \mathrm{C}$, the exposure solution was carefully decanted and the parasites were rinsed twice with seawater before being transferred to plastic Petri dishes holding fresh seawater. Animals were allowed to recover for $24 \mathrm{~h}$ in a $12^{\circ} \mathrm{C}$ incubator before their attachment and motility behaviour was rated. Behavioural responses were evaluated blind by an observer unaware of strain affiliation and exposure history. Rating was according to viability criteria "live" (normal behaviour), "weak", "moribund" and "dead", as defined in an earlier study ${ }^{37}$ that slightly modified earlier definitions ${ }^{38,39}$. Parasites were considered unaffected when rated "live" or "weak" and affected when rated "moribund" or "dead". All loA00 parasites exposed to $2 \mu \mathrm{g} \mathrm{L}^{-1}$ deltamethrin for $30 \mathrm{~min}$ followed by $24 \mathrm{~h}$ of incubation in seawater were rated "dead". In all other groups, maximally $10 \%$ of parasites were deemed "affected" (data not shown). Only individuals deemed unaffected were collected for RNA extraction and subsequent determination of transcript abundance.

\subsection{Quantitative RT-PCR of L. salmonis $\mathrm{Na}_{v}$ homologues}

Quantitative reverse transcription polymerase chain reaction (RT-qPCR) was used to determine the abundance of $\mathrm{Na}_{v} 1.1, \mathrm{Na}_{v} 1.2$ and $\mathrm{Na}_{v} 1.3$ transcripts. Oligonucleotide primers for target and reference genes (ribosomal subunit 40S, 40S; elongation factor 1-alpha, ef1a and hypoxanthine-guanine phosphoribosyltransferase, hgprt) (Additional file 2, Table S2) ${ }^{40}$ were used at $300 \mu \mathrm{M}$ with 1/160 of the cDNA synthesis reaction (2.5 $\mu \mathrm{L}$ of a 1:20 dilution) and $5 \mu \mathrm{L}$ of SYBR-green qPCR mix (ABgene, UK) in a total volume of $10 \mu \mathrm{L}$. Reactions were run in a Mastercycler RealPlex ${ }^{2}$ (Eppendorf, UK) ( $\left.n=6\right)$. Amplifications were carried out including negative controls containing no cDNA (NTC, no template control) and controls omitting reverse transcriptase enzyme (-RT) to ascertain the absence of DNA contamination. 
Thermal cycle and melting curves were performed as described previously ${ }^{41}$. The size of PCR products was checked by agarose gel electrophoresis along with appropriate markers and the correct identity of amplicons was confirmed by sequencing a random subset of samples. Relative quantification of transcript expression was achieved by including on each PCR plate a parallel set of reactions containing serial dilutions of a pool of all experimental cDNA samples, allowing to derive for each sample the estimated relative copy number of the transcript of interest, corrected for the efficiency of the reaction. The normalised expression values were generated by the $\Delta \Delta \mathrm{Ct}$ method ${ }^{42}$ and the results expressed as mean normalised ratios ( $\pm S E$ ) between the RUs of target genes and a reference gene index calculated from the geometric mean of the threshold cycles of the three most stable reference genes (i.e. 40S, ef1a and hgprt) (Supporting information, Table S3) ${ }^{43}$.

\subsection{Data analysis and statistical tests}

Transcript expression was presented as the relative expression ratio of each gene (relative units, RUs). Effects of sex/stage and parasite strain on transcript expression were assessed by two-way ANOVA for each of the sodium channels. The effects of deltamethrin exposure on sodium channel transcription of parasites of the same sex/stage and strain were then assessed by one-way ANOVA, followed by post-hoc comparisons to the control group using Dunnett's test. When performing one-way ANOVAs, a Bonferroni correction was applied to significance levels to keep the experiment-wise type I error rate within the overall alpha level. Channel sequences, obtained by Sanger sequencing of RT-PCR products, were compared between $L$. salmonis strains IoA-00 and IoA-02 by performing the Freeman-Halton extension of the Fisher exact probability test ${ }^{44}$ for a $2 \times 3$ table for each polymorphic nucleotide site, i.e. comparing the frequencies of three possible outcomes for each individual parasite (sequence variant 1 , sequence variant 2 , or double peak) between the two parasite strains tested. After genotyping parasites for SNP loci, allele frequencies were compared among strains using Fisher's method as implemented in the program genepop version $4.2^{45}$. The significance level was $p<0.05$ for all statistical tests performed.

\section{Results}

\section{1 $\mathrm{Na}_{v} 1$ homologues in L. salmonis}

Genomic screening of voltage-gated sodium channels identified three L. salmonis $\mathrm{Na}_{v} 1$ homologues (Supporting information, Table $S 1$ ), referred to in this study as $L s N a_{v} 1.1$, $L s a_{v} 1.2$ and $L s a_{v} 1.3$, following to the accepted nomenclature for $\mathrm{Na}_{v}$ channels ${ }^{11}$. Transcript sequences of the three channels identified in a multi-stage transcriptome of $L$. 
salmonis ${ }^{30}$ yielded cDNA sequences covering the whole open reading frame (ORF) for $L s a_{v} 1.1$ and $L s N a_{v} 1.3$ and a partial sequence for $L s N a_{v} 1.2$ (Supporting information, Table S1). The deduced amino acid sequences of the identified channels contain typical features present in all $\mathrm{Na}_{\mathrm{v}} 1^{2,31}$, such as four homologous domains (I-IV), the sodium sensing amino acids "DEKA", and the inactivation gate motif ("IFM" in rat $\mathrm{Na}_{v} 1.2$; "MFM" or "AFM" in $L$. salmonis $\mathrm{Na}_{\mathrm{v}} 1$ homologues) (Supporting information, Figure $\mathrm{S} 1$ ).

\subsection{Phylogenetic analysis}

Phylogenetic analyses were performed on sequences of conserved domain I of arthropod $\mathrm{Na}_{v} 1$ channels (Figure 2). In the obtained tree, sequences cluster together according to taxonomic groups, such as insects, malacostracans, copepods and arachnids. Copepod homologues formed two separate clusters. $L s N a_{v} 1.1$ grouped closely with the sequence of the planktonic copepod $A$. hudsonica and one of three $C$. finmarchicus sodium channels, whereas $L s \mathrm{Na}_{v} 1.2$ and $L s N a_{v} 1.3$ grouped more distantly together with two remaining $C$. finmarchicus homologues (Figure 2).

\subsection{Channel nucleotide sequences in resistant and susceptible parasites}

$\mathrm{Na}_{v} 1$ channels comprise four internally homologous domains (I-IV) that each contain six $\alpha-$ helical transmembrane segments (S1-S6) connected by intracellular loops ${ }^{31}$. Kdr type resistance shows a recessive mode of inheritance in other arthropods ${ }^{31}$ and is associated with mutations in specific regions of domains I-III, namely segments S5 and S6 and the linker helix connecting S4 and S5. For channels $N a_{v} 1.1, N a_{v} 1.2$ and $N a_{v} 1.3$, domains I to III were amplified by RT-PCR in both susceptible (IoA-00) and pyrethroid-resistant (IoA-02) salmon lice ( $n \geq 5$ animals per strain) and sequenced. While a number of sequence polymorphisms were identified for genes $L s N a_{v} 1.1$ and $L s N a_{v} 1.2$, their frequencies did not differ significantly between loA-00 and loA-02 salmon lice (Supporting information, Tables S4-5). In contrast, sequences at two polymorphic sites in $L_{s N a} 1.3$ were significantly affected by parasite strain origin (Supporting information, Table S6). The nucleotide polymorphism a3947c corresponds to a missense mutation, causing an amino acid change located in the intracellular loop between DII and DIII, while g4465a represents a synonymous mutation at a site in IIIS4 of the channel (counts according to HACA01028321.1). Moreover, strain differences were apparent but not significant $(P=0.061)$ for a non-synonymous mutation at a site in IIS5 of $L s N a_{v} 1.3$ (a3041g, Table S6).

For $L s N a_{v} 1.3$ mutations that showed a trend towards sequence differences between loA-00 and loA-02 ( $P<0.1$, Table S6), potential association with deltamethrin resistance was further investigated by assessing the genotypes of a greater number of parasites in four strains, using allele-specific PCR assays. Deltamethrin susceptible strains loA-00 and loA-01 as well 
as deltamethrin resistant strains loA-02 and loA-03 were considered at $n=14$ to 16 parasites per strain (Table 1). For the SNP marker a3041g, allele frequencies differed significantly between all possible pairings of deltamethrin resistant and susceptible strains, and between resistant strains loA-02 and loA-03 (Table 2). Allele frequencies at SNP loci a3947c and g4465a did not significantly differ among strains IoA-00, IoA-01 and IoA-03, but were significantly different in strain loA-02 as compared to the other studied strains (Table 2).

\subsection{Transcript expression of $L$. salmonis voltage-gated Na channels}

L. salmonis are sexually dimorphic, with the development of parasites from the hatching of eggs to reaching the adult stage taking longer to complete in females than males. In synchronised cohorts of developing parasites, preadult II females and adult males appear at the about the same time and are similar in size. For this reason, these stages are routinely used for bioassays in our laboratory. In order to make findings of this study comparable to bioassay data, preadult II females and adult male sea lice, obtained from synchronised cohorts of strains IoA-00 and IOA-02, were used in studies of transcript expression of paralogous $\mathrm{Na}_{v}$ genes (Figure 3). For each $\mathrm{Na}_{v}$ channel, two way ANOVA was used to assess whether transcription differed between the loA-00 and loA-02 strains and between preadult II females and adult males. Transcript abundance was not affected by strain origin for any of the studied $\mathrm{Na}_{v}$ genes but differed significantly between adult males and preadult Il females for all three channels $(P<0.001)$. Moreover, there was a significant interaction between strain origin and sex/stage effects on the transcription of $N a_{v} 1.2$ and $N a_{v} 1.3(P<$ 0.05). When $\mathrm{Na}_{v}$ gene transcription was studied after a 30 min exposure to deltamethrin followed by $24 \mathrm{~h}$ recovery, deltamethrin $\left(0.05 \mu \mathrm{g} \mathrm{L}^{-1}\right)$ significantly decreased $N a_{v} 1.3$ transcription $(P<0.05)$ in loA-00 adult males (Figure 3 ). 


\section{Discussion}

The present study characterised voltage-gated sodium channels in the fish parasite L. salmonis, in which pyrethroid resistance is common ${ }^{20}$. The results revealed the existence of three voltage-gated sodium channel paralogues in $L$. salmonis that were named $L s N a_{v} 1.1$, $L s \mathrm{Na}_{v} 1.2$ and $L s \mathrm{Na}_{v} 1.3$ according to the accepted nomenclature ${ }^{11,46}$. The predicted salmon louse $\mathrm{Na}_{\mathrm{v}} 1$ polypeptides are $\sim 40 \%$ identical to vertebrate $\mathrm{Na}_{\mathrm{v}} 1$ subunits and comprise four highly conserved homologous domains (I-IV), paralleling the architecture of all known voltage-gated sodium channels ${ }^{1,2}$. Each domain contains six hydrophobic transmembrane segments (S1-S6) including the voltage sensor in the S4 segments, characterised by positively charged arginine amino acid residues. A re-entrant loop between S5 and S6 embedded into the transmembrane region of the channels was identified, known to form the $\mathrm{Na}^{+}$selective filter (SF) constituted by four amino acids (Asp, Glu, Lys and Ala, [DEKA]) ${ }^{47}$. The presence of a lysine $(K)$ in the third position has proven crucial for $\mathrm{Na}^{+}$permeability ${ }^{48}$. Kdr-type resistance of arthropods against pyrethroids and DDT results from point mutations of $\mathrm{Na}_{v} 1$ leading to amino acid changes of the channel ${ }^{2,49-51}$. Since the initial identification of $\mathrm{Na}_{v} 1$ mutations in $k d r$ and super-kdr strains of the housefly ${ }^{52,53}$, many kdr-type mutations have been reported from resistant isolates of different arthropod species ${ }^{2}$, with key mutations having evolved independently in different arthropod species ${ }^{54}$. The comparative functional characterisation of wild type $\mathrm{Na}_{v} 1$ and selected channels with kdr mutations following recombinant expression in Xenopus oocytes revealed that many kdr-type mutations decrease the pyrethroid affinity of $\mathrm{Na}_{v} 1^{2}$. Moreover, functional studies of $\mathrm{Na}_{v} 1$ mutants showed that some single amino acid substitutions have synergistic effects when present in combination ${ }^{2}$. Interestingly, kdr mutations cluster in particular channel regions, such as the linker between S4 and S5 (L45) and S5 of DI, L45, S5 and S6 of DII, and S6 of DIII $^{2}$, suggesting $\mathrm{Na}_{v} 1$ may possess discrete pyrethroid binding sites. Potential $\mathrm{Na}_{v} 1$-ligand interactions were assessed through the construction of homology models of insect $\mathrm{Na}_{\mathbf{v}} 1$ based on available crystal structures of bacterial sodium and mammalian potassium channels ${ }^{55,56}$. This approach led to the prediction of two pyrethroid binding sites, one of which involves L45 and S5 of DII and S6 of DIII, while the second site is composed of L45 and S5 of DI and S6 of DII ${ }^{57,58}$.

In order to identify $\mathrm{kdr}$ in the present study, L. salmonis $\mathrm{Na}_{v} 1$ sequences were amplified from a restricted number of individuals $(n=5-6)$ of two strains (IoA-00, drug susceptible; loA-02, highly pyrethroid resistant) and sequenced. Using this approach three candidate nucleotide polymorphisms potentially associated to resistance were identified in $L s N_{v} 1.3$ (a3041g, a3947c and a4456g), while no potential kdr mutations were found in $L s N a_{v} 1.1$ and $L s N a_{v} 1.2$. 
The $L s N a_{v} 1.3$ candidate markers were then further assessed by genotyping a larger number of individuals ( $n=14-16)$ in the above and two additional strains. Data obtained with the nonsynonymous a3041g mutation supported a putative association between deltamethrin resistance and the expression of the $3041 \mathrm{~g}$ allele, which was present in the deltamethrin resistant strains studied, IoA-02 and loA-03, but not detected in the deltamethrin susceptible strains IoA-00 and loA-01. In contrast, genotyping further individuals and taking into account further parasite strains failed to confirm an association of single nucleotide polymorphisms a3947c and a4456g with deltamethrin resistance.

The non-synonymous a3041g mutation results in the substitution of an isoleucine by a valine at amino acid position 1014 of $\mathrm{LsNa}_{\mathrm{v}} 1.3$ (numbering according to predicted amino acid sequence of GenBank accession number HACA01028321.1). When numbered according to Musca domestica Vssc1 (GenBank accession number: AAB47604), this mutation corresponds to $1936 \mathrm{~V}$ located in S5 of DII (Figure 1, Figure S1), a conserved channel region contributing to one of the two predicted pyrethroid binding sites and known to contain kdr mutations $^{2}$. Interestingly, a homologous mutation has been identified in the $\mathrm{Na}_{v} 1$ sequence of pyrethroid resistant corn earworm, Helicoverpa zea (Boddie) (Lepidoptera: Noctuidae) (1951 V, also corresponding to I936V of M. domestica Vssc1), where it was found in the cDNA but not gDNA sequence, suggesting its introduction by RNA editing ${ }^{59}$. In contrast, the a3041g mutation was apparent in L. salmonis in both cDNA and gDNA sequences (this study). Although isoleucine and valine are amino acids with similar physiochemical properties, the substitution within one of the two predicted pyrethroid-binding sites of the channel corresponds to a position of $\mathrm{Na}_{v} 1$ where valine is the residue found in vertebrates, while isoleucin is found in the wild-type channel of arthropods. O'Reilly et al. ${ }^{58}$ suggested that this difference in sequence could contribute to species-dependent binding affinity of pyrethroids to $\mathrm{Na}_{v} 1$, and subsequently differences in toxicity. Furthermore, the isoleucine to valine mutation in the $\mathrm{Na}_{v} 1$ gene in Drosophila melanogaster was confirmed to cause decreased pyrethroid sensitivity after cloning into in the Xenopus oocyte system and subsequent electrophysiological analysis ${ }^{60}$.

An earlier study assessed $\mathrm{Na}_{v} 1.1$ sequences in L. salmonis sampled from different salmon farming sites ${ }^{23}$. A non-synonymous mutation in $L s N a_{v} 1.1$, which leads to a glutamine to arginine change at a position corresponding to amino acid 945 of the Musca domestica $\mathrm{Na}_{v} 1$, was present in 10 to $67 \%$ of $L$. salmonis of populations collected at fish farms where decreased efficacy of pyrethroids had been reported, and absent in parasite populations from sites with no reports of pyrethroid treatment failures ${ }^{23}$. While the Q945R mutation maps to a $\mathrm{Na}_{v} 1$ region harbouring known kdr mutations, no direct evidence exists that the mutation affects $\mathrm{Na}_{v} 1$ affinity to pyrethroids ${ }^{23}$. In a later study on individually selected pyrethroid 
resistant salmon lice from four locations in Norway ${ }^{61}$, neither this, nor other pyrethroidassociated mutations were found in $L s N a_{v} 1.1$ from adult females (Supporting information, Table S7).

A recent study demonstrated a sex-biased pattern of inheritance of pyrethroid resistance in L. salmonis, studying strains from Scottish sites also investigated in the present study ${ }^{26}$. In reciprocal crosses between pyrethroid resistant loA-02 salmon lice and susceptible loA-00 parasites, the F1 and F2 progeny of all families derived from a deltamethrin susceptible P0 male and a deltamethrin resistant P0 female were resistant ${ }^{26}$. In contrast, in families derived from crosses of the inverse orientation, F1 animals were susceptible and, depending on the family, $0-20 \%$ of $\mathrm{F} 2$ parasites were resistant. Maternal inheritance of deltamethrin resistance in L. salmonis has also been demonstrated in a separate study on Norwegian salmon lice strains $^{25}$. In the Scottish study, deltamethrin resistant strains isolated from different regions of Scotland showed nearly identical mitochondrial haplotypes ${ }^{26}$. The results suggested a major contribution of mitochondrial genetic factors to pyrethroid resistance in L. salmonis; however the presence of the $20 \%$ resistant F2 parasites in some families initiated from an IoA-02 male and an loA-00 female suggests that nuclear genetic determinants may be responsible for the additional observed resistance. Kdr resistance in other arthropods is a recessive trait ${ }^{4}$ and such a mode of inheritance would be consistent with the observed resistance phenotype in the $\mathrm{F} 2$ crosses ${ }^{26}$.

In mammals, multiple $\mathrm{Na}_{v} 1$ paralogues have arisen from gene duplications and chromosomal rearrangements, and differ in channel gating kinetics, ontogeny and tissue expression profiles, with certain isoforms primarily involved in the nervous response while others are majorly expressed in the skeletal muscle or the heart ${ }^{31,46}$. In contrast, in insects multiple $\mathrm{Na}_{v}$ isoforms are typically produced through mechanisms of alternative splicing 31,34,62. For instance, in Drosophila, one $\mathrm{Na}_{v} 1$ gene exhibits multiple alternative exons that can be used to produce 29 splice types, and although no pharmacological tests have been performed to determine specific drug and toxin affinity, the functional characterisation revealed clear differences in the channel kinetics ${ }^{62}$.

Relatively little is known about $\mathrm{Na}_{v} 1$ genes in crustaceans. A single $\mathrm{Na}_{v} 1$ gene has been found in the genome of the cladoceran Daphnia ${ }^{34}$. In contrast, the copepod C. finmarchicus possesses a diverse $\mathrm{Na}_{v} 1$ subfamily with at least three isoforms ${ }^{34,63}$, paralleling the findings obtained with L. salmonis in the present study. Planktonic free-living copepods, believed to be the most abundant metazoans in the oceans ${ }^{64}$, are integral to marine food webs ${ }^{65}$. Thus, multiplicity of $\mathrm{Na}_{\mathrm{v}}$ channel function could be related to the adaptation of copepods to a variety of stressors, e.g. exposure to algal toxins targeting $\mathrm{Na}_{v} 1^{63}$. The presence of different $\mathrm{Na}_{v} 1$ homologues in $L$. salmonis could further be related to subfunctionalisation, such as 
different types of neurons expressing different $\mathrm{Na}_{v} 1$ paralogues. The fact that a kdr mutation was observed in $L s \mathrm{Na}_{v} 1.3$, but not $L s N a_{v} 1.1$ and $L s \mathrm{Na}_{v} 1.2$, could point to a greater importance of $L s \mathrm{Na}_{v} 1.3$ for survival during the host-attached phase of the life cycle. More research is needed to resolve functions of $\mathrm{Na}_{v} 1$ paralogues in L. salmonis.

In the present study, relative expression of $L$. salmonis $\mathrm{Na}_{v} 1$ transcripts showed a similar pattern of transcription for $L s \mathrm{Na}_{v} 1.2$ and $L s \mathrm{Na}_{v} 1.3$, for both of which transcript expression was low in preadult II females and high in adult males. In contrast, the $L s N a_{v} 1.1$ transcript was more abundant in preadult II females as compared to adult males of the same age. In $L$. salmonis male reproductive behaviours include mate searching and testing, formation of precopulatory complexes with preadult-II females and mate guarding ${ }^{66}$. Copulation with the guarding male occurs typically directly after the moult of the female to the final adult stage, and involves the male cementing a pair of spermatophores onto the female's genital complex, blocking further insemination ${ }^{67}$. However, using microsatellite markers, multiple paternity of offspring of individual females was demonstrated, providing evidence for polyandry in L. salmonis ${ }^{68}$. In view of the marked contrasts in reproductive behaviours between the sexes, it may be speculated that sexually differential $N a_{v} 1$ transcription could reflect sex specific neuronal adaptations.

\section{Conclusion}

Findings of the present study indicate that a kdr-mutation in $L s N a_{V} 1.3$ may contribute to deltamethrin resistance in $L$. salmonis.

\section{Acknowledgements}

This study was supported by United Kingdom Biotechnology and Biological Sciences Research Council grant BB/L022923/1 awarded to Armin Sturm. The authors gratefully acknowledge additional financial support from the Scottish Salmon Producer's Organisation to the aforementioned project. This study was further supported by the Norwegian Research Council through the Centre for Research-based Innovation, Sea Lice Research Centre, grant numbers NFR 203513, awarded to Tor E. Horsberg and NFR 280306, awarded to Marit J. Bakke, and a PhD studentship co-funded by PHARMAQ/Zoetis awarded to Claudia Tschesche. The authors further gratefully acknowledge funding received from the MASTS pooling initiative (The Marine Alliance for Science and Technology for Scotland). MASTS is funded by the Scottish Funding Council (grant reference HR09011) and contributing institutions. The funders had no role in study design, data collection and analyses, decision to publish, or preparation of the manuscript. 


\section{References}

1 Catterall WA, Cellular and molecular biology of voltage-gated sodium channels., Physiol Rev 72:S15-48 (1992).

2 Dong K, Du Y, Rinkevich F, Nomura Y, Xu P, Wang L, et al., Molecular biology of insect sodium channels and pyrethroid resistance., Insect Biochem Mol Biol 50:1-17, Elsevier Ltd (2014).

3 Catterall WA, Cestèle S, Yarov-Yarovoy V, Yu FH, Konoki K, and Scheuer T, Voltage-gated ion channels and gating modifier toxins, Toxicon 49:124-141 (2007).

Davies TGE, Field LM, Usherwood PNR, and Williamson MS, DDT, pyrethrins, pyrethroids and insect sodium channels., IUBMB Life 59:151-162 (2007).

Rinkevich FD, Du Y, and Dong K, Diversity and convergence of sodium channel mutations involved in resistance to pyrethroids, Pestic Biochem Physiol 106:93-100, Elsevier Inc. (2013).

Soderlund DM, Resmethrin, the first modern pyrethroid insecticide, Pest Manag Sci 71:801807 (2015).

Sparks TC, Insecticide discovery: An evaluation and analysis, Pestic Biochem Physiol 107:817, Elsevier Inc. (2013).

Zhu F, Lavine L, O'Neal S, Lavine M, Foss C, and Walsh D, Insecticide Resistance and Management Strategies in Urban Ecosystems, Insects 7:2, Multidisciplinary Digital Publishing Institute (2016).

9 Ffrench-Constant RH, Daborn PJ, and Le Goff G, The genetics and genomics of insecticide resistance., Trends Genet 20:163-170 (2004).

10 Popp J, Peto K, and Nagy J, Pesticide productivity and food security. A review, Agron Sustain Dev 33:243-255 (2013).

Goldin AL, Evolution of voltage-gated Na+ channels, J Exp Biol 584:575-584 (2002).

Martins AJ and Valle D, The Pyrethroid Knockdown Resistance, Insecticides - Basic and Other Applications, InTech, Rijeka, pp. 17-38 (2012).

13 Edi C V, Djogbénou L, Jenkins AM, Regna K, Muskavitch M a T, Poupardin R, et al., CYP6 $\mathrm{P} 450$ enzymes and $A C E-1$ duplication produce extreme and multiple insecticide resistance in the malaria mosquito Anopheles gambiae., PLoS Genet 10:e1004236 (2014).

14 Ffrench-Constant $\mathrm{RH}$, The molecular genetics of insecticide resistance, Genetics 194:807-815 (2013).

15 Torrissen O, Jones S, Asche F, Guttormsen A, Skilbrei OT, Nilsen F, et al., Salmon lice-impact on wild salmonids and salmon aquaculture., J Fish Dis 36:171-194 (2013).

16 Johnson SC, Treasurer JW, Bravo S, and Nagasawa K, A review of the impact of parasitic copepods on marine aquaculture, Zool Stud 43:229-243 (2004). 
17 Skern-Mauritzen R, Torrissen O, and Glover KA, Pacific and Atlantic Lepeophtheirus salmonis (Krøyer, 1838) are allopatric subspecies: Lepeophtheirus salmonis salmonis and L. salmonis oncorhynchi subspecies novo., BMC Genet 15:32 (2014).

18 Costello MJ, Ecology of sea lice parasitic on farmed and wild fish., Trends Parasitol 22:475483 (2006).

19 Burridge L, Weis JS, Cabello F, Pizarro J, and Bostick K, Chemical use in salmon aquaculture: A review of current practices and possible environmental effects, Aquaculture 306:7-23, Elsevier B.V. (2010).

Aaen SM, Helgesen KO, Bakke MJ, Kaur K, and Horsberg TE, Drug resistance in sea lice: a threat to salmonid aquaculture, Trends Parasitol 31:72-81, Elsevier Ltd (2015).

Sevatdal S, Copley L, Wallace C, Jackson D, and Horsberg TE, Monitoring of the sensitivity of sea lice (Lepeophtheirus salmonis) to pyrethroids in Norway, Ireland and Scotland using bioassays and probit modelling, Aquaculture 244:19-27 (2005).

Helgesen KO, Bravo S, Sevatdal S, Mendoza J, and Horsberg TE, Deltamethrin resistance in the sea louse Caligus rogercresseyi (Boxhall and Bravo) in Chile: bioassay results and usage data for antiparasitic agents with references to Norwegian conditions., J Fish Dis 37:877-890 (2014).

Fallang A, Denholm I, Horsberg TE, and Williamson MS, Novel point mutation in the sodium channel gene of pyrethroid-resistant sea lice Lepeophtheirus salmonis (Crustacea: Copepoda)., Dis Aquat Organ 65:129-136 (2005).

Sevatdal S, Fallang A, Ingebrigtsen K, and Horsberg TE, Monooxygenase mediated pyrethroid detoxification in sea lice (Lepeophtheirus salmonis)., Pest Manag Sci 61:772-778 (2005).

Bakke MJ, Agusti C, Bruusgaard JC, and Sundaram AYM, Deltamethrin resistance in the salmon louse , Lepeophtheirus salmonis (Krøyer): Maternal inheritance and reduced apoptosis, Sci Rep 8:8450 (2018).

Carmona-Antoñanzas G, Bekaert M, Humble JH, Boyd S, Roy W, Bassett DI, et al., Maternal inheritance of deltamethrin resistance in the salmon louse Lepeophtheirus salmonis (Krøyer) is associated with unique mtDNA haplotypes, PLoS One 12:e0180625 (2017).

27 Carmona-Antoñanzas G, Humble JL, Carmichael SN, Heumann J, Christie HRL, Green DM, et al., Time-to-response toxicity analysis as a method for drug susceptibility assessment in salmon lice, Aquaculture 464:570-575, Elsevier B.V. (2016).

28 Heumann J, Carmichael S, Bron JE, Tildesley A, and Sturm A, Molecular cloning and characterisation of a novel P-glycoprotein in the salmon louse Lepeophtheirus salmonis., Comp Biochem Physiol C Toxicol Pharmacol 155:198-205, Elsevier Inc. (2012).

29 Chen L, Zhang H, Finiguerra M, Bobkov Y, Bouchard C, Avery DE, et al., A novel mutation from gene splicing of a voltage-gated sodium channel in a marine copepod and its potential 
effect on channel function, J Exp Mar Bio Ecol 469:131-142 (2015).

Carmona-Antoñanzas G, Carmichael SN, Heumann J, Taggart JB, Gharbi K, Bron JE, et al., A survey of the ATP-binding cassette $(A B C)$ gene superfamily in the salmon louse (Lepeophtheirus salmonis), ed. by Corsi I, PLoS One 10:e0137394 (2015).

Yu FH and Catterall WA, Overview of the voltage-gated sodium channel family., Genome Biol 4:207, BioMed Central (2003).

Sievers F, Wilm A, Dineen D, Gibson TJ, Karplus K, Li W, et al., Fast, scalable generation of high-quality protein multiple sequence alignments using Clustal Omega, Mol Syst Biol 7:539, School of Medicine and Medical Science, UCD Conway Institute of Biomolecular and Biomedical Research, University College Dublin, Dublin, Ireland. (2011).

Stamatakis A, RAxML version 8: a tool for phylogenetic analysis and post-analysis of large phylogenies., Bioinformatics 30:1312-1313 (2014).

Lenz PH, Roncalli V, Hassett RP, Wu L-S, Cieslak MC, Hartline DK, et al., De novo assembly of a transcriptome for Calanus finmarchicus (Crustacea, Copepoda) - The dominant zooplankter of the north Atlantic Ocean, ed. by lanora A, PLoS One 9:e88589, Public Library of Science (2014).

Myakishev M V, Khripin Y, Hu S, and Hamer DH, High-Throughput SNP Genotyping by AlleleSpecific PCR with Univeral Energy-Transfer-Labeled Primers, Genome Res 11:163-169 (2001).

Blanquer A, Phylogéographie intraspécifique d'un poisson marin, le flet Platichthys flesus L. (Heterosomata): polymorphisme des marqueurs nucléaires et mitochondriaux, Montpellier 2, France (1990).

Igboeli OO, Fast MD, Heumann J, and Burka JF, Role of P-glycoprotein in emamectin benzoate (SLICE ${ }^{\circledR}$ ) resistance in sea lice, Lepeophtheirus salmonis, Aquaculture 344-349:4047, Elsevier B.V. (2012).

Westcott JD, Stryhn H, Burka JF, and Hammell KL, Optimization and field use of a bioassay to monitor sea lice Lepeophtheirus salmonis sensitivity to emamectin benzoate., Dis Aquat Organ 79:119-131 (2008).

Sevatdal S and Horsberg TE, Determination of reduced sensitivity in sea lice (Lepeophtheirus salmonis Krøyer) against the pyrethroid deltamethrin using bioassays and probit modelling, Aquaculture 218:21-31 (2003).

40 Carmichael SN, Bron JE, Taggart JB, Ireland JH, Bekaert M, Burgess STG, et al., Salmon lice (Lepeophtheirus salmonis) showing varying emamectin benzoate susceptibilities differ in neuronal acetylcholine receptor and GABA-gated chloride channel mRNA expression, BMC Genomics 14:408 (2013). 
of dietary phospholipid requirement of Atlantic salmon, Salmo salar, fry, Biochim Biophys Acta - Mol Cell Biol Lipids 1851:1428-1441 (2015).

Pfaffl MW, Quantification strategies in real-time PCR, ed. by Bustin SA, A-Z of Quantitative PCR, Biotechnology Series, International University Line, La Jolla, CA, pp. 87-112 (2004). Pfaffl MW, Tichopad A, Prgomet C, and Neuvians TP, Determination of stable housekeeping genes, differentially regulated target genes and sample integrity: BestKeeper--Excel-based tool using pair-wise correlations., Biotechnol Lett 26:509-515 (2004).

Freeman $\mathrm{GH}$ and Halton $\mathrm{JH}$, Note on exact treatment of contingency, goodness of fit and other problems of significance, Biometrika 38:141-149 (1951).

Rousset F, Genepop'007: a complete reimplementation of the Genepop software for Windows and Linux, Mol Ecol Resour 8:103-108 (2008).

Catterall WA, Goldin AL, and Waxman SG, International Union of Pharmacology. XLVII. Nomenclature and Structure-Function Relationships of Voltage-Gated Sodium Channels, Pharmacol Rev 57:397-409 (2005).

Favre I, Moczydlowski E, and Schild L, On the structural basis for ionic selectivity among Na+, $\mathrm{K}+$ and Ca2+ in the voltage-gated sodium channel, Biophys J 71:3110-3125 (1996).

Heinemann S, Terlau H, Stühmer W, Imoto K, and Numa S, Calcium channel characteristics conferred on the sodium channel by single mutations, Nature 356:441-443 (1992).

Bricelj VM, Connell L, Konoki K, MacQuarrie SP, Scheuer T, Catterall WA, et al., Sodium channel mutation leading to saxitoxin resistance in clams increases risk of PSP, Nature 434:763-767, Nature Publishing Group (2005).

Jost MC, Hillis DM, Lu Y, Kyle JW, Fozzard HA, and Zakon HH, Toxin-resistant sodium channels: parallel adaptive evolution across a complete gene family., Mol Biol Evol 25:10161024, Oxford University Press (2008).

Weston DP, Poynton HC, Wellborn GA, Lydy MJ, Blalock BJ, Sepulveda MS, et al., Multiple origins of pyrethroid insecticide resistance across the species complex of a nontarget aquatic crustacean, Hyalella azteca, Proc Natl Acad Sci 110:16532-16537 (2013).

(1)

Williamson MS, Martinez-Torres D, Hick CA, and Devonshire AL, Identification of mutations in the housefly para-type sodium channel gene associated with knockdown resistance (kdr) to pyrethroid insecticides, Mol Genet Genomics 252:51-60 (1996).

Miyazaki M, Ohyama K, Dunlap DY, and Matsumura F, Cloning and sequencing of the paratype sodium channel gene from susceptible and kdr-resistant German cockroaches (Blattella germanica) and house fly (Musca domestica), Mol Gen Genet 252:61-68 (1996).

Rinkevich FD, Du Y, and Dong K, Diversity and convergence of sodium channel mutations involved in resistance to pyrethroids, Pestic Biochem Physiol 106:93-100, Elsevier Inc. (2013). 
55 Payandeh J, Scheuer T, Zheng N, and Catterall WA, The crystal structure of a voltage-gated sodium channel, Nature 475:353-358, Nature Publishing Group (2011). (2006).

Du YZ, Nomura Y, Satar G, Hu ZN, Nauen R, He SY, et al., Molecular evidence for dual pyrethroid-receptor sites on a mosquito sodium channel, Proc Natl Acad Sci U S A 110:11785-11790 (2013).

O'Reilly AO, Khambay BPS, Williamson MS, Field LM, Wallace BA, and Davies TGE, Modelling insecticide-binding sites in the voltage-gated sodium channel, Biochem J 396:255263 (2006).

Hopkins BW and Pietrantonio P V., The Helicoverpa zea (Boddie) (Lepidoptera: Noctuidae) voltage-gated sodium channel and mutations associated with pyrethroid resistance in fieldcollected adult males, Insect Biochem Mol Biol 40:385-393, Elsevier Ltd (2010).

Usherwood PNR, Davies TGE, Mellor IR, O'Reilly AO, Peng F, Vais H, et al., Mutations in DIIS5 and the DIIS4-S5 linker of Drosophila melanogaster sodium channel define binding domains for pyrethroids and DDT, FEBS Lett 581:5485-5492 (2007).

Helgesen KO, Monitoring of drug resistance and resistance development mechanisms in salmon lice (Lepeophtheirus salmonis), Norwegian University of Life Sciences, Adanstuen, Norway (2015).

O'Donnel Olson R, Liu Z, Nomura Y, Song W, and Dong K, Molecular and functional characterization of voltage-gated sodium channel variants from Drosophila melanogaster, Insect Biochem Mol Biol 38:604-610 (2008).

Finiguerra M, Avery DE, and Dam HG, Determining the advantages, costs, and trade-offs of a novel sodium channel mutation in the copepod Acartia hudsonica to paralytic shellfish toxins (PST), ed. by Ai T, PLoS One 10:e0130097, Public Library of Science (2015).

Humes AG, How many copepods?, Hydrobiologia 292-293:1-7, Kluwer Academic Publishers (1994).

Banse K, Zooplankton: Pivotal role in the control of ocean production, ICES J Mar Sci 52:265277, Oxford University Press (1995).

66 Ritchie G, Mordue (Luntz) AJ, Pike AW, and Rae GH, Observations on mating and reproductive behaviour of Lepeophtheirus salmonis, Krøyer (Copepoda: Caligidae), J Exp Mar Bio Ecol 201:285-298 (1996).

67 Ritchie G, Mordue AJ, Pike AW, and Rae GH, Morphology and ultrastructure of the reproductive system of Lepeophtheirus salmonis (Krøyer, 1837) Copepoda: Caligidae), J Crustac Biol 16:330-346 (1996). 
Lepeophtheirus salmonis despite complex precopulatory and postcopulatory mate-guarding, Mar Ecol Prog Ser 303:225-234 (2005). 
Table 1. Genotyping of different $L$. salmonis strains at SNP loci within $L s N a_{v} 1.3$. Individuals from deltamethrin (DM) susceptible (S) and resistant (S) strains were subjected to allele specific PCR genotyping. Per strain, 6-8 animals of each sex were tested. Data were pooled within strains as no significant sex-specific allelic or genotypic differentiation was found. Genotype frequencies in the different strains showed no significant deviation from Hardy-Weinberg equilibrium.

\begin{tabular}{|c|c|c|c|c|c|c|c|c|}
\hline \multicolumn{9}{|c|}{ Genotypes (\%) } \\
\hline $\begin{array}{l}\text { SNP } \\
\text { locus }\end{array}$ & Strain & $\begin{array}{c}\text { DM } \\
\text { susceptibility }\end{array}$ & $\mathbf{N}$ & gg & ga & aa & $\begin{array}{l}\text { Frequency } \\
\text { allele } 1\end{array}$ & $\begin{array}{l}\text { Frequency } \\
\text { allele } 2\end{array}$ \\
\hline \multirow[t]{4}{*}{ a3041g } & IoA-00 & $S$ & 16 & 0.00 & 0.00 & 100.00 & 0.000 & 1.000 \\
\hline & IoA-01 & S & 16 & 0.00 & 0.00 & 100.00 & 0.000 & 1.000 \\
\hline & IoA-02 & $\mathrm{R}$ & 15 & 60.00 & 40.00 & 0.00 & 0.800 & 0.200 \\
\hline & IoA-03 & $\mathrm{R}$ & 14 & 21.43 & 28.57 & 50.00 & 0.357 & 0.643 \\
\hline \multirow[t]{4}{*}{ a3947c } & IoA-00 & S & 16 & 6.25 & 62.50 & 31.25 & 0.375 & 0.625 \\
\hline & IoA-01 & S & 16 & 6.25 & 43.75 & 50.00 & 0.281 & 0.719 \\
\hline & IoA-02 & $\mathrm{R}$ & 15 & 0.00 & 0.00 & 100.00 & 0.000 & 1.000 \\
\hline & IoA-03 & $\mathrm{R}$ & 14 & 7.14 & 14.29 & 78.57 & 0.143 & 0.857 \\
\hline \multirow[t]{4}{*}{ a4456g } & IoA-00 & S & 16 & 31.25 & 62.50 & 6.25 & 0.625 & 0.375 \\
\hline & IoA-01 & S & 16 & 50.00 & 43.75 & 6.25 & 0.719 & 0.281 \\
\hline & IoA-02 & $\mathrm{R}$ & 15 & 100.00 & 0.00 & 0.00 & 1.000 & 0.000 \\
\hline & IoA-03 & $\mathrm{R}$ & 14 & 78.57 & 14.29 & 7.14 & 0857 & 0.143 \\
\hline
\end{tabular}


Table 2. Pairwise allelic differentiation between $L$. salmonis strains at three SNP loci. The table shows P-values of pairwise comparisons of allele frequencies between deltamethrin susceptible (S) and resistant $(\mathrm{R})$ strains using the exact G-test, based on the data shown in Table 1. Allele frequencies are regarded significantly different between strains when the P-value is lower than 0.05 (given in bold print).

\begin{tabular}{llrrr} 
SNP locus & Strains & IoA-01 (S) & IoA-02 (R) & IoA-03 (R) \\
\hline a3041g & IoA-00 (S) & 1.00000 & $<\mathbf{0 . 0 0 0 1}$ & $<\mathbf{0 . 0 0 0 1}$ \\
& IoA-01 (S) & & $<\mathbf{0 . 0 0 0 1}$ & $\mathbf{0 . 0 0 0 1 4}$ \\
\multirow{3}{*}{ a3947c } & IoA-02 (R) & & & $\mathbf{0 . 0 0 1 2 7}$ \\
& IoA-00 (S) & 0.58604 & $\mathbf{0 . 0 0 0 2 6}$ & 0.15480 \\
& IoA-01 (S) & & $\mathbf{0 . 0 0 1 6 7}$ & 0.38541 \\
& IoA-02 (R) & & & $\mathbf{0 . 0 2 0 1 6}$ \\
a4456g & IoA-00 (S) & 0.79582 & $\mathbf{0 . 0 0 0 1 2}$ & 0.08039 \\
& IoA-01 (S) & & $\mathbf{0 . 0 0 0 2 9}$ & 0.13766 \\
& IoA-02 (R) & & & $\mathbf{0 . 0 4 9 1 0}$ \\
\hline
\end{tabular}




\section{Figure Legends}

Figure 1. Voltage-gated sodium channel $\left(\mathrm{Na}_{v} 1\right)$ domain structure. Positions marked with * denote the location of previously found kdr mutations in arthropod species, the effect of which on channel pyrethroid susceptibility was confirmed in Xenopus oocytes expression studies $^{2}$. The white star indicates the position of the non-synonymous single nucleotide polymorphism a3041g in LsNav1.3, corresponding to the point mutation I936V in the predicted amino acid sequence of the channel (numbering according to Musca domestica Vssc1).
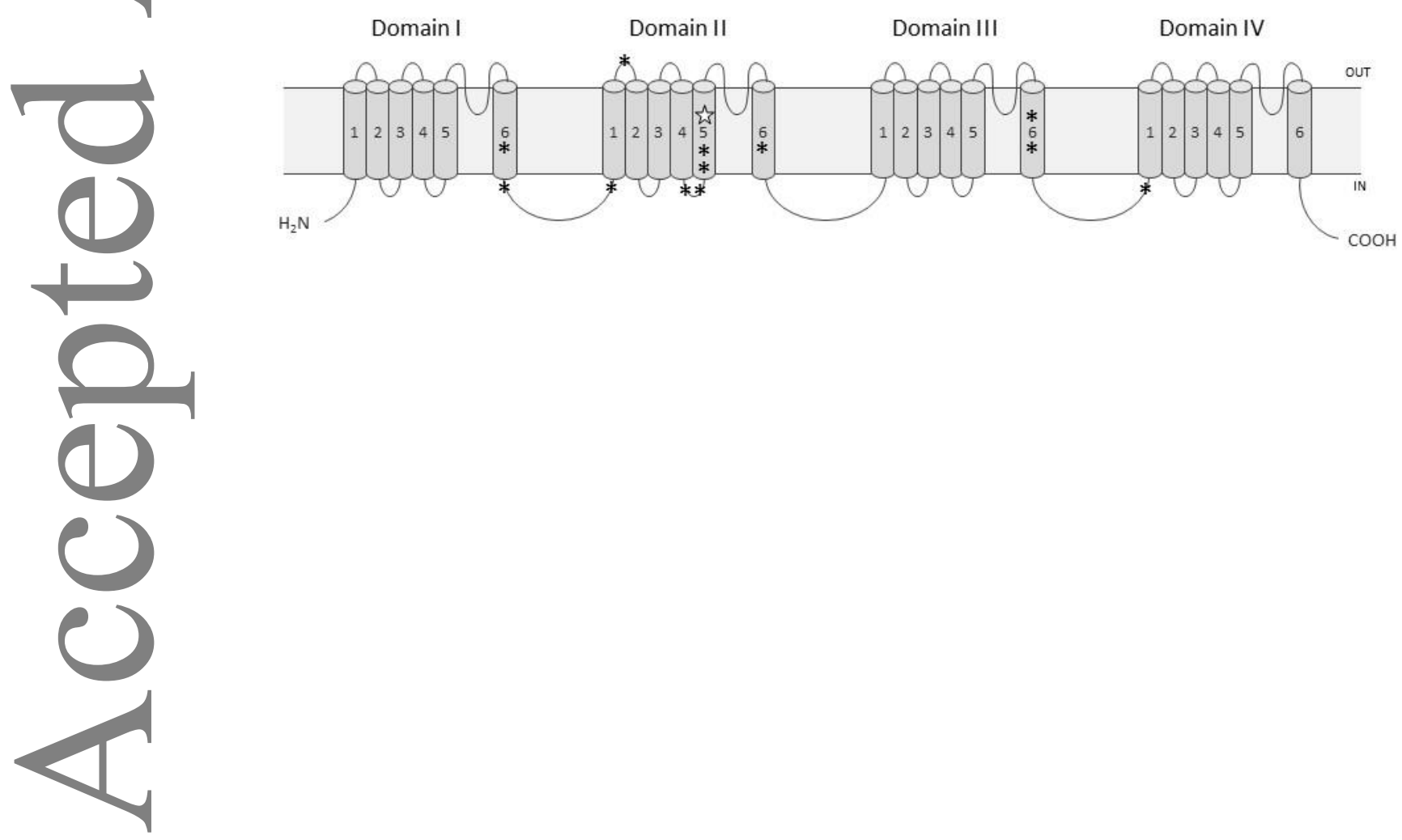
Figure 2. Phylogenetic analysis of voltage-gated sodium channels $\mathrm{Na}_{v} 1$ in L. salmonis. The tree was constructed based on the amino acid sequences of domain I of $\mathrm{Na}_{v} 1$ paralogues identified in a salmon louse transcriptome ${ }^{29}$ subjected to a maximum likelihood analysis using RAxML ${ }^{33}$. For phylogenetic reconstruction, the WAG substitution model of rate heterogeneity among sites were implemented. Numbers at the basal nodes represent the frequencies with which the presented tree topology was obtained after bootstrapping (1000 iterations). The scale bar represents 0.2 amino-acid substitutions per site.

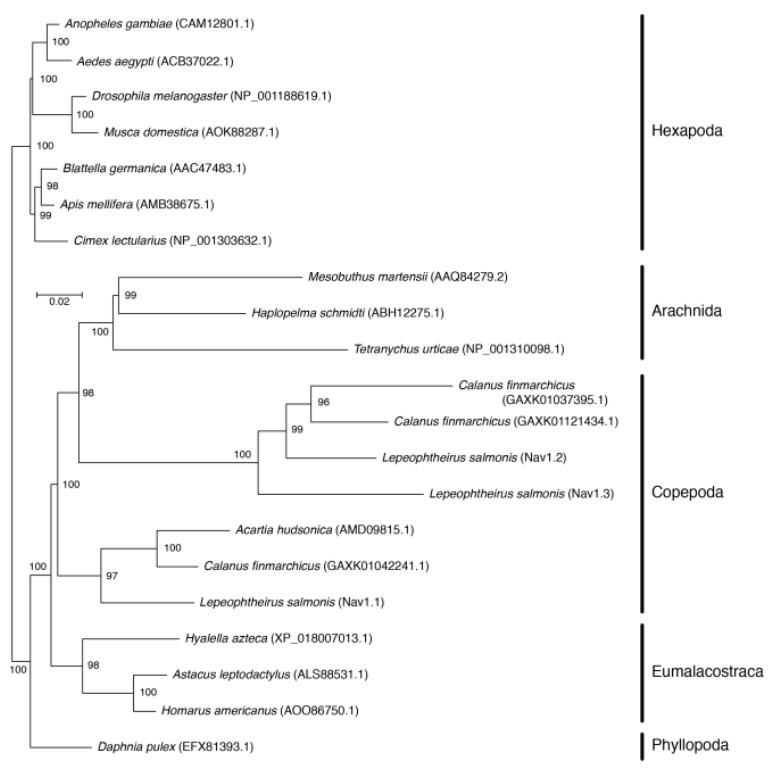

Figure 3. Expression of voltage-gated sodium channels $\mathrm{Na}_{v} 1$ in L. salmonis preadult II females and adult males. Transcript expression was expressed as relative units (RUs) calculated from the mean normalised ratios $(n=6, \pm S E)$ between the estimated copy numbers of target genes and the estimated copy numbers of the reference genes. Columns represent the normalised expression values determined in parasites of different strains (IoA00, drug-susceptible; IoA-02 pyrethroid-resistant) and instar after $30 \mathrm{~min}$ of treatment with the indicated level of deltamethrin or solvent vehicle PEG300 (controls) and $24 \mathrm{~h}$ of recovery. In treatments labelled with a dagger $(\dagger)$, all parasites were rated moribund or dead and therefore excluded from analyses. Asterisks $\left(^{*}\right)$ indicate a significant treatment effect on channel transcription when compared to control animals of the same strains and sex/instar (One-way ANOVA and Dunnett's test; $P<0.05$ after Bonferroni correction). 

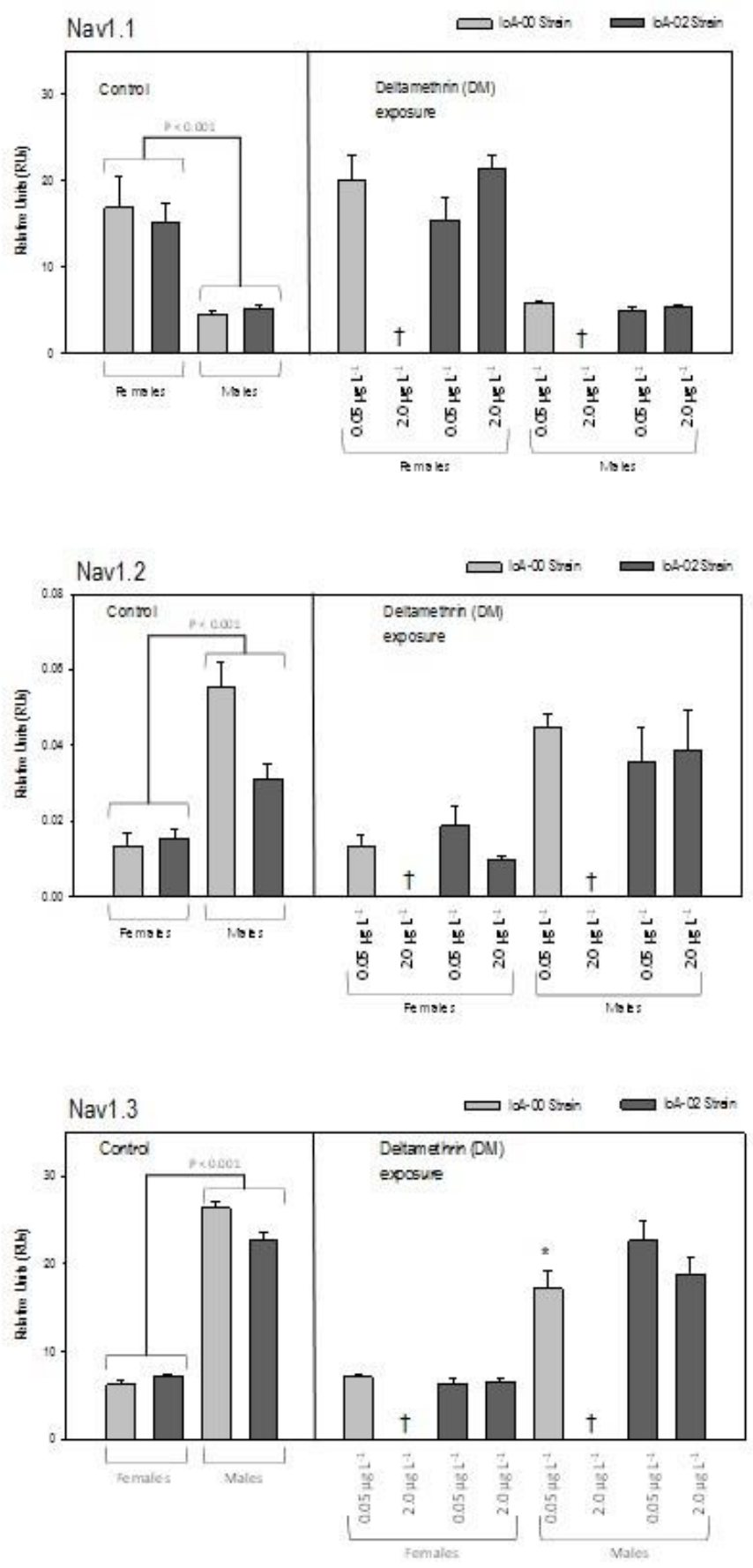